\title{
Modelling the airborne infection risk of tuberculosis for a research facility in eMalahleni, South Africa
}

\author{
Ralf R. Küsel, ${ }^{1}$ Ian K. Craig, ${ }^{1^{\star}}$ and Anton C. Stoltz ${ }^{2}$
}

Objective: The development of a detailed mathematical modelling framework for the risk of airborne infectious disease transmission in indoor spaces. This allows for detailed mathematical analysis of experiments conducted at the Airborne Infections Research (AIR) facility, eMalahleni, South Africa.

Methods: The mathematical modelling builds on the commonly used Wells-Riley equation by including additional mechanisms and dynamics, and is presented modularly. This modelling framework was applied to an experiment conducted at the AIR facility from 31 August 2015 to 4 December 2015, in which the efficacy of upper room germicidal ultraviolet (GUV) irradiation as an environmental control was tested. The upper room GUV irradiation efficacy experiment in the facility did not produce expected outcomes of having fewer infections in the test animal room than the control room, and several potential reasons for this are investigated using the simulated model.

Results: Simulation results indicate that dynamic effects, caused by switching the GUV lights, power outages, or introduction of new patients, did not result in the unexpected outcomes; the experiment design sufficiently catered for these situations. However, a sensitivity analysis highlights that significant uncertainty exists with risk of transmission predictions based on current measurement practises, due to the reliance on large viable literature ranges for parameters.

Significance: The developed mathematical modelling framework is found useful in exploring possible causes of why an experiment at the AIR facility did not produce expected results.

KEY WORDS: airborne infectious disease transmission, biological system modelling, Mycobacterium tuberculosis, parameter extraction, sensitivity analysis

\section{INTRODUCTION}

Quantifying the risk of transmission of airborne infectious diseases is useful in the evaluation of the effectiveness of an infection control strategy. As an infection control strategy, rapid active case finding

\footnotetext{
${ }^{1}$ Department of Electrical, Electronic and Computer Engineering, University of Pretoria, Pretoria, South Africa ${ }^{2}$ Department of Internal Medicine, University of Pretoria, Pretoria, South Africa

Address correspondence to Ian K. Craig, Department of Electrical, Electronic and Computer Engineering, University of Pretoria, Pretoria, South Africa: ian.craig@up.ac.za
}

of infected individuals and community intervention is the more effective method (Nardell and Dharmadhikari, 2010; Yuen et al., 2015; Nardell, 2016). However, active case finding is much more expensive than the alternative passive case finding (Mupere et al., 2013). Therefore, environmental control, such as ventilation and germicidal ultraviolet (GUV) lights, remains an important consideration for the reduction of the risk of transmission of an airborne infectious disease (Beggs et al., 2000; Li et al., 2007). Environmental factors are especially important in the time between infection and diagnosis of the infection (Dharmadhikari et al., 2014). 
A mathematical model can serve as an effective tool to quantify the risk of transmission of airborne infectious diseases (Sze To and Chao, 2010). Mathematical models not only help in understanding, but have the advantage that an exploration of a theoretical concept through mathematical simulation is typically quicker and cheaper than conducting an experiment (Jeffrey et al., 2003; Craig and Xia, 2005). Mathematical models are an important part of the design of intelligent systems, where quantitative models help to gain a deeper understanding of the process involved and allow for systematic design of appropriate responses to the problem (Antsaklis, 1994).

Tuberculosis (TB) remains a severe health threat in the world, despite advances in treatment and diagnosis in the past decade (Lönnroth et al., 2010). In South Africa, especially, there is a call to improve the public health response (Karim et al., 2009). Of significant importance in the reduction of incidence is the interruption of transmission (Dye et al., 2013; Yates et al., 2016). The disease is caused by the Mycobacterium tuberculosis ( $M$. $t b$ ) pathogen, and the main method of transmission is via aerosoled droplets that are expelled through the respiratory tract of a host (Schwander and Ellner, 2006).

This paper provides a framework for the inclusion of a number of different factors into a single model of the risk of TB transmission in indoor spaces, specifically for the situation of an airborne infectious disease research facility. The modelling framework is applied to the risk of transmission of $M$. tuberculosis in an airborne infections research (AIR) facility in eMalahleni, Mpumalanga, South Africa (Dharmadhikari et al., 2011, 2012, 2014; Mphaphlele et al., 2015). The model simulation results are compared with the results from an experiment conducted at the research facility.

\section{MODEL DEVELOPMENT}

In the study of airborne infectious diseases, animals are often used as a method of detecting the risk to humans (Dharmadhikari and Nardell, 2008). Human to guinea pig transmission is a common method that is used to study air disinfection techniques (Riley et al., 1962, 1959; Nardell, 2004; Escombe et al., 2007; Dharmadhikari et al., 2011). This usually requires the sentinel animal to be placed in a different room to the source of the pathogen, with mechanical ventilation used as a proxy to the risk of infection in the ward.
The Wells-Riley equation (Riley, 1974) remains the most commonly used model to quantify the risk of transmission in indoor spaces (Sze To and Chao, 2010). However, the Wells-Riley equation is limited to situations in which the infected individuals are in the same zone as the susceptible individuals (Noakes and Sleigh, 2008). This makes the Wells-Riley model not ideal for the modelling of airborne infectious disease research facilities, where the sentinel animals are not in the same airspace.

The approach to model development, followed by this paper, is one of defining multiple different effects of the risk of transmission within indoor spaces as individual mathematical descriptions. These mathematical descriptions can then be combined. Where the mechanisms are linear, the law of additivity is applicable; where the mechanisms are nonlinear, an alternative approach is given. This allows freedom to construct relevant models for different situations and research questions.

The model will be given in a deterministic, mechanistic, nonlinear state-space and continuous time format. The deterministic model represents an average expected risk of transmission, which is assumed to be the same as the average of an equivalent stochastic model. The deterministic model is chosen as opposed to a stochastic probabilistic model (Nicas and Seto, 1997) or a Markov chain model (Nicas, 2000), which simplifies the modelling process by removing the need to specify the uncertainty through the definition of random variable distributions in the model.

The probability that the pathogen deposits on the individual's alveoli follows a Poisson distribution (Wells, 1955; Riley et al., 1978). This probability distribution is described through an exponential function, similar to the result of solving a linear differential equation. Since other influencing factors on the risk of transmission can be expressed in terms of rates of change, this allows for the use of differential equations to describe the model.

A convenient way in which to represent a model is the state-space format that relates the inputs and state variables through simultaneous, first-order differential equations. An advantage of the statespace format is its relevance in control systems theory, which can assist in the study of infectious diseases(Craig and Xia, 2005; Doyle et al., 2007; du Toit and Craig, 2015). Methods for parameter extraction are well developed in control systems theory, and provide tools for determining unknown 
parameters in a model from measurements (Xia, 2003; Xia and Moog, 2003; Filter et al., 2005). The Gammaitoni-Nucci model also follows a statespace format (Gammaitoni and Nucci, 1997) and, in contrast to the Wells-Riley equation, allows for the consideration of non-steady state conditions of airborne infectious particles (Beggs et al., 2003).

Four different categories of state variables are defined for the model framework used in this paper; however, in the construction of a model, the number of state variables within each category may vary. The first category is the groups of susceptible individuals that represent the populations with the potential of being infected. The second and third categories of state variables are the groups of infected individuals and the groups of exposed individuals. The distinction between the infected and the exposed population classifications depends on the definition of infection used in the model, and whether an incubation period is included in the model (Noakes et al., 2006). The final category of state variables considered, is the number of infectious aerosol particles within a space.

Parameters in the model will be given as nontime varying to avoid confusion between parameters and state variables, although the parameters may be implemented as functions of time.

\subsection{New infections in the susceptible populations}

New infections in the susceptible population $S_{i}$, are described as an average probabilistic reduction of the susceptible population based on the expected exposure to the pathogen. Susceptible populations that are geographically separated can be defined as populations in different zones and denoted with their subscript $i$. A zone is an air space, which can be a room or parts of a room (Nicas, 1996b; Noakes and Sleigh, 2008).

The rate of new infections is proportional to the pulmonary ventilation rate $p_{i}\left(m^{3} \cdot s^{-1}\right)$ of the susceptible population and the concentration of infectious particles that the susceptible population is potentially exposed to (Gammaitoni and Nucci, 1997). The concentration of infectious particles is given as the fraction of the number of infectious particles $C_{i}(t)$ (quanta) over the volume $V_{i}\left(m^{3}\right)$. An infectivity term $\theta_{i}\left(\right.$ quanta $\left.^{-1}\right)$ allows for the consideration of a fraction of the re-breathed air that is inhaled, but does not cause infection, where, for example, not all of the inhaled pathogens land on alveoli (Nicas et al., 2005). The infectivity term also addresses the dimensionality problem of the WellsRiley equation, even if it is taken as unity (Sze To and Chao, 2010).

New infections in the susceptible populations are described mathematically, in this model framework, by:

$$
\frac{d S_{i}(t)}{d t}=-p_{i} \theta_{i} \frac{C_{i}(t)}{V_{i}} S_{i}(t)
$$

\subsection{Infections without an incubation period}

New infections, when no incubation period is considered, are treated as the increase of infected population due to the average probabilistic reduction of the susceptible population based on the expected exposure to the pathogen. The rate at which the infected population grows due to exposure to the pathogen is given by

$$
\frac{d I_{i}(t)}{d t}=p_{i} \theta_{i} \frac{C_{i}(t)}{V_{i}} S_{i}(t) .
$$

Equation 2 is the complement of Equation 1. This ensures the total population size remains constant when no external factors, such as birth or deaths within the population, are considered. It is assumed viable to leave out the consideration of these when modelling an airborne infectious diseases research facility.

\subsection{Infections with an incubation period}

The incubation period of the disease is important to consider when the measurement of the risk is done using a sentinel animal. To include an incubation period, an extra epidemiological population is introduced as another state variable, namely the exposed individuals population category (Noakes et al., 2006). The exposed individuals are a hypothetical state, where the individual has been infected, but is asymptotic.

If an incubation period is considered, then the individuals first become exposed, before they transition to the infected state. The exposed population then increases due to the average probabilistic reduction of the susceptible population based on the expected exposure to the pathogen, instead of the infected population. The rate at which the exposed population grows due to exposure to the pathogen is given by

$$
\frac{d E_{i}(t)}{d t}=p_{i} \theta_{i} \frac{C_{i}(t)}{V_{i}} S_{i}(t)
$$


The transition from the exposed to the infected state is based on a incubation period delay rate $\alpha_{i}\left(d a y^{-1}\right)$. The terms governing this mechanism in the exposed and the infected individuals, is given as

$$
\frac{d E_{i}(t)}{d t}=-\alpha_{i} E_{i}(t)
$$

in the exposed individuals population, and

$$
\frac{d I_{i}(t)}{d t}=\alpha_{i} E_{i}(t)
$$

in the infected individuals population.

\subsection{Generation of infectious particles}

Infectious particles are assumed to be generated in the airspace through the expulsion of small, pathogen containing aerosol particles by infected individuals (Ehrenkranz and Kicklighter, 1972; Fiegel et al., 2006; Tang et al., 2006), with the infectivity of an airborne disease taken to be directly linked to these cough generated aerosols (Fennelly et al., 2004; Jones-López et al., 2013).

The infectious particles in a zone $C_{i}(t)$ are modelled in units quanta. A quantum of infection is defined as the number of infectious particles that would infect $63.2 \%$ (i.e. $1-e^{-1}$ ) of the population, if every member of the population was exposed to this quantity of infectious particles (Wells, 1955). The quanta is a hypothetical infectious dose unit and describes the infectivity as well as the infectious source strength of an epidemiological outbreak (Sze To and Chao, 2010).

The total average size distribution of coughed droplet nuclei is approximately 0.5-6 $\mu \mathrm{m}$ (Yang et al., 2007) and there is a proven increase of aerosol particles, in the region of microbial particle sizes, of 3-4 $\mu \mathrm{m}$ due to human room occupation (Bhangar et al., 2014). Therefore, a direct link between the number of infected and the quantity of infectious particles in an indoor space seems reasonable.

The rate at which infectious particles are generated in a zone is proportional to the rate of quanta expelled by an individual $\phi_{i}\left(\right.$ quanta $\left.\cdot h^{-1}\right)$. If the infectious individuals $I_{i}(t)$ in a zone are each assumed to generate quanta at the same rate on average, then the rate at which quanta in a zone is generated is given by

$$
\frac{d C_{i}(t)}{d t}=\phi_{i} I_{i}(t)
$$

The quanta generation rate per infected individual $\phi$ is backwards calculated from epidemiological data. The quanta generation rate per infected individual is in the range of $1.25-30840$ quanta $\cdot h^{-1}$, depending on the situation (Nardell et al., 1991; Gammaitoni and Nucci, 1997; Beggs et al., 2003); however, for an average TB patient, the range is between 1.25-12.7 quanta $\cdot h^{-1}$ (Nardell et al., 1991). Inherent in the backwards calculation of the quanta generation rate is the viability loss and the deposition loss of the pathogen, therefore these mechanisms should not be considered twice when using the quanta of infection (Fisk et al., 2004; Sze To and Chao, 2010).

However, the quanta of infection does not have to be used as the unit of infectious particles, and $\phi$ could be set equal to an equivalent dose response function. An example of this is to set

$$
\phi=G \beta \quad,
$$

where $G$ is the emission rate of pathogens per infected individual, and $\beta$ the alveoli disposition fraction (Nicas, 1996a). However, when doing so, the implications of moving from a hypothetical infectious dose unit (quanta) to an actual infectious dose unit need to be carefully considered. The viability of the aerosol pathogen, for instance, would then need to be taken into account.

\subsection{Dispersion and distribution of infectious particles}

The proximity of infected individuals to susceptible individuals, in relation to the ventilation air ducts, can be an important factor (Li et al., 2007). In situations where the air in the room is not wellmixed, this can be modelled by using different zones and the transfer rate of infectious particles between these zones (Nicas, 1996b, 2000; Noakes and Sleigh, 2008).

The physical law of conservation of mass is used as the modelling principle for the dispersion of infectious particles through air movement. This implies that infectious particles in a zone are assumed to stay in that zone unless they are removed through air movement or decay from that zone. The removal of infectious particles from a zone due to air movement is given by

$$
\frac{d C_{i}(t)}{d t}=-\frac{Q_{i}}{V_{i}} C_{i}(t)
$$

where the function $Q_{i}\left(m^{3} \cdot s^{-1}\right)$ describes the airflow out of the zone in volume of air per unit time. The 
environment is assumed to be an unmodelled sink of the pathogens.

The increase of infectious particles in zone $i$ due to the removal of infectious particles from another zone, $j$, is then given by

$$
\frac{d C_{i}(t)}{d t}=\frac{Q_{j}}{V_{j}} C_{j}(t) \quad, \text { where } j \neq i .
$$

Equation 9 assumes that $Q_{j}(t)\left(m^{3} \cdot s^{-1}\right)$ is the airflow out of zone $j$, into zone $i$. The model indirectly accounts for temperature changes between zones through the consideration of volumetric airflow, because the rate at which infectious particles are moved between zones is directly proportional to the volumetric airflow rates. Volumetric airflow rates are proportional to temperature conditions according to Charles's law, which forms part of the ideal gas law (Chapman and Cowling, 1970).

These equations for the dispersion and diffusion of infectious particles do not account for the dynamics involved in the dispersion of infectious particles from infected individuals into the zone through coughing, speaking and other respiratory expulsions (King et al., 2013; Li et al., 2013; Mousavi and Grosskopf, 2014).

\subsection{Removal of infectious airborne particles using GUV lights}

The removal of infectious particles due to GUV irradiation will be assumed manifested as an exponential decay of the bacteria, with none of the bacteria being resistant to the irradiation (Nazaroff et al., 1998; Kowalski et al., 2000; Miller and MacHer, 2000; Fisk et al., 2004). This is then modelled as

$$
\frac{d C_{i}(t)}{d t}=-k_{i} H_{i} \frac{V_{i U}}{V_{i}} C_{i}(t)
$$

where $k_{i}$ is the standard decay rate constant due to GUV light for the microbe considered and $H_{i}$ is the UV fluence $\left(\mu W \cdot \mathrm{cm}^{-2}\right)$ of the fixture (Kowalski et al., 2000). The range of the standard decay rate constant $k$ is between $0.0987-0.4721 \mathrm{~cm}^{2} \cdot s \cdot \mu W^{-1}$ for M. tuberculosis (David, 1973; Riley et al., 1976). The volume that is irradiated by the GUV fixture in zone $i$ is given by $V_{i U}$.

\section{EMALAHLENI AIR FACILITY MODEL}

As an example of how this modelling framework can be used, a model will be developed for the Airborne Infections Research (AIR) facility at eMalahleni (formerly known as Witbank),
Mpumalanga (Dharmadhikari et al., 2011, 2012; Mphaphlele et al., 2015). Experimental data that was obtained from a study, on the effectiveness of upper room GUV irradiation, conducted at the AIR facility, is then compared to model predictions. The study at the AIR facility was run from 31 August 2015 to 4 December 2015.

The AIR facility in eMalahleni consists of wards for TB patients and two animal rooms, based on the design of the research facility used by Riley from 1958-1962 (Riley, 2001). The wards consist of three rooms, with two beds in each room, patient ablutions and a patient day room. A maximum of six patients can be accommodated in the AIR facility. A layout of the ward area and the patient rooms is shown in Figure 1.

The assumption in the model will be made that the air is well-mixed, because paddle fans in each of the ward rooms help to ensure the air mixing in the room. The air turnover rate of the paddle fans used in the AIR facility is approximately $57 h^{-1}$ (Mphaphlele et al., 2015). This is much greater than the ventilation rate, which was $3-6 A C \cdot h^{-1}$. This means that for each air change that occurs, the air in the room is circulated approximately 10 times.

The facility is described by two sets of susceptible populations $S_{i}(t)$, representing the guinea pigs in each animal room, and a single population of infectious individuals $I_{w}(t)$. Three zones are considered in this model. The first zone considered is the ward $i=w$. The other two zones considered is the space occupied by the cages in each of the two animal rooms; $i=1$ for animal room one and $i=2$ for animal room two. Since the guinea pigs only

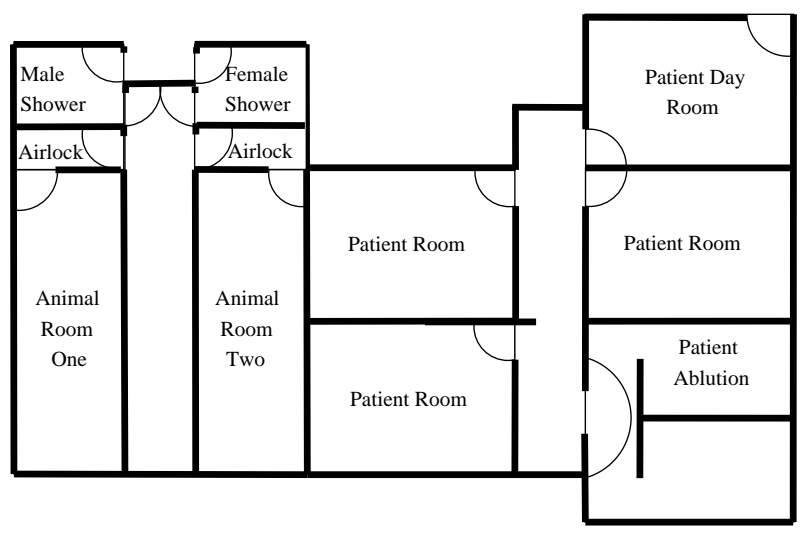

Fig. 1. The layout of the wards and animal rooms of the airborne infections research (AIR) facility in eMalahleni. 
have access to the space that their cage occupies, only this space will be considered as a zone. The volume considered in each of these zones is given by $V_{i}$. There are no paddle fans in the animal rooms, therefore the air that the guinea pigs are exposed to is only moved through the ventilation system. However, the ventilation air into the animal room is split up and delivered through an installed diffuser in front of each of the cages. This system ensures that the sentinel animals in their cages are each exposed to approximately equal amounts of ventilation air.

Nine state variables are used to describe the AIR facility. Three states describe the number of infectious particles in each of the three zones $C_{i}(t)$. Six states describe the guinea pig populations; three for each animal room: $i=1$ for animal room one and $i=2$ for animal room two. The guinea pig population is described through classification as infected $I_{i}(t)$, exposed $E_{i}(t)$ or susceptible $S_{i}(t)$. The model is given in 11-19.

$$
\begin{aligned}
\frac{d S_{1}(t)}{d t} & =-p \theta \frac{C_{1}(t)}{V_{1}} S_{1}(t) \\
\frac{d S_{2}(t)}{d t} & =-p \theta \frac{C_{2}(t)}{V_{2}} S_{2}(t) \\
\frac{d E_{1}(t)}{d t} & =p \theta \frac{C_{1}(t)}{V_{1}} S_{1}(t)-\alpha E_{1}(t) \\
\frac{d E_{2}(t)}{d t} & =p \theta \frac{C_{2}(t)}{V_{2}} S_{2}(t)-\alpha E_{2}(t) \\
\frac{d I_{1}(t)}{d t} & =\alpha E_{1}(t) \\
\frac{d I_{2}(t)}{d t} & =\alpha E_{2}(t) \\
\frac{d C_{w}(t)}{d t} & =\phi_{w} I_{w}-\frac{Q_{w}}{V_{w}} C_{w}(t) \\
\frac{d C_{1}(t)}{d t} & =\frac{Q_{1 \text { in }}}{V_{w}} C_{w}(t)-\frac{Q_{1 \text { out }}}{V_{1}} C_{1}(t) \\
\frac{d C_{2}(t)}{d t} & =\frac{Q_{2 \text { in }}}{V_{w}} C_{w}(t)-\frac{V_{2 \text { out }}}{V_{2}} C_{2}(t)
\end{aligned}
$$

Patients, as the source of the infectious particles $I_{w}$, are recruited for the study, based on referral by the adjoining multi-drug resistant (MDR) TB hospital. This study was approved by the human studies committees of the South African MRC, the US CDC, and the Harvard School of Public Health. Only individuals with lab confirmed positive acid- fast bacilli (AFB) sputum smear test results are considered for inclusion in the study. The patients are intended to remain at the AIR facility for two weeks. The timeline of the patients admitted at the AIR facility for the study is given in Figure 2. It will be assumed that the generation of quanta is representative of an average, constant value $\phi_{w}$ for all infected individuals.

By using the theoretical quanta of infection, the uncertainty of a number of factors can be lumped into the variable $\phi$, allowing for easier estimation of this parameter in the face of uncertainty. The quanta of infection does not, however, give a realistic indication of the number of infectious particles in a zone, but was chosen, instead of a dose response model, because of the problem of a lack of infectious dose data for infections in humans (Sze To and Chao, 2010).

Each animal room contained 90 guinea pigs, which served as a sentinel animal model for the risk of airborne transmission of TB. Outbred, specific pathogen free, Dunkin-Hartley guinea pigs were used in the experiment, with an equal number being male and female. Two guinea pigs are kept in a cage, but each animal room has 50 cages, leaving 10 cages unused per animal room. It is assumed that all the guinea pigs have the same constant average pulmonary respiratory rate $p=p_{1}=p_{2}$ and that they are equally susceptible to infection $\theta=\theta_{1}=\theta_{2}$. The incubation period of the pathogen is given by $\alpha=\alpha_{1}=\alpha_{2}$, the time taken from infection until the guinea pig exhibits symptoms of infection or reacts positively to tuberculin skin test (TST) diagnosis. Infected animals are removed and not replaced during the course of an experiment.

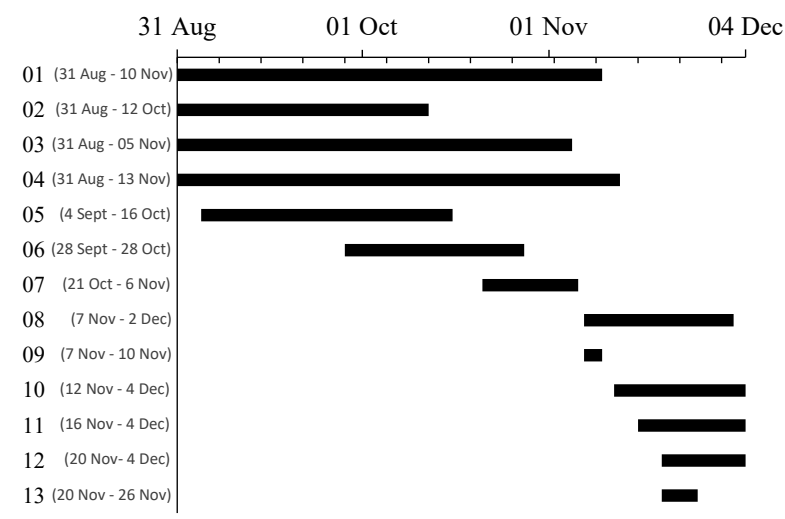

Fig. 2. The timeline for the patients admitted to the AIR facility and the duration of their admittance. 
Animal care was overseen by a licensed laboratory veterinarian and all protocols were approved by the Animal Use committees of the South African MRC, the US CDC, and Harvard Medical School.

The ventilation system airflow out of the wards $Q_{w}$ is routed to either of the two animal rooms. The animal rooms receive ventilation air from either the patient ward $Q_{i \text { in }}$ or high efficiency particulate arrestance (HEPA) filtered outside air on alternate days. The airflow out of the animal rooms $Q_{i \text { out }}$ is assumed to be equal to the airflow into an animal room, because the airflow out is controlled to maintain a constant negative pressure in the animal rooms.

On the days that animal room one receives the ward air, upper room GUV lights are turned on inside the wards. The standard decay rate constant due to GUV irradiation for $M$. tuberculosis is given by $k_{w}$. Animal room two is assumed to be the control room for this experiment aimed at determining the efficacy of upper room GUV light.

The two animal rooms in the facility are maintained under animal biosafety level 3 conditions (World Health Organization, 2004). The temperature in the animal rooms is kept at $22 \pm 1^{\circ} \mathrm{C}$, with a relative humidity of $50 \pm 10 \%$. Workers are required to wear a full face respirator, and must shower before entering and upon exiting the animal room area. The showers and airlocks are indicated in Figure 1.

\section{STUDY SIMULATION}

The AIR facility study that ran from 31 August 2015 to 4 December 2015 is simulated using the model (11) - (19). The simulation is performed by solving the model using the Runga-Kutta integration method with a fixed time step of 1 minute per time step (Shampine, 1994; Shampine and Reichelt, 1997). The ward, duct and animal cage dimensions are obtained from measurements at the facility and given in Table I .

The ventilation data and the GUV fixture switching are obtained from the Supervisory Control and Data Acquisition (SCADA) system installed the AIR facility. This gives a real-time measurement of the airflow rates between the different zones of the facility. The ventilation airflow rate data from the SCADA system for the airflow out of the ward is given in Figure 3 and the measured airflow into and out of the two animal rooms is given in Figure 5 .

The upper room GUV fixture fluence is $5 \mu \mathrm{W}$. $\mathrm{cm}^{-2}$ when it is on (Mphaphlele et al., 2015), and the fluence is taken as zero when it is off. The GUV light is assumed to uniformly irradiate the space in the patient ward above a height of $2.1 \mathrm{~m}$, or a volume of $V_{w U}=21.7 \mathrm{~m}^{3}$. The on and off switching data is obtained from the SCADA system, and the resulting GUV fluence in the ward is shown in Figure 4.

The infectious source parameter $I_{w}$ is defined by the number of patients at the AIR facility and is obtained from the patient timeline, Figure 2.

A guinea pig is taken to be infected when its TST result is positive. The measure of the number of infected guinea pigs is shown in Table III .

The remaining model parameters needed for the simulation are taken from literature. The source of these parameters is given in Table II , along with the literature reference. Where the given parameters

Table I . The measured parameters from the AIR facility used for the simulation. The parameters are defined in their most convenient units.

\begin{tabular}{lc} 
Measurement & Value \\
\hline$A_{\text {duct:ward out }}$ & $0.09 \mathrm{~m}^{2}$ \\
$A_{\text {duct:ward-animal room }}$ & $0.015 \mathrm{~m}^{2}$ \\
$A_{\text {duct:animal room clean air }}$ & $0.034 \mathrm{~m}^{2}$ \\
$N_{\text {cage }}$ & 50 \\
$V_{\text {ward room } 1}$ & $3.0 m \times 4.8 m \times 2.6 m$ \\
$V_{\text {ward room } 2}$ & $3.2 m \times 4.8 m \times 2.6 m$ \\
$V_{\text {ward room } 3}$ & $2.9 m \times 4.8 m \times 2.6 m$ \\
$V_{\text {ward common room }}$ & $5 m \times 4 m \times 2.6 m$ \\
$V_{\text {cage }}$ & $0.56 m \times 0.35 m \times 0.36 m$ \\
$V_{w}$ & $164.8 m^{3}$ \\
$V_{U w}$ & $21.7 m^{3}$ \\
$V_{a 1}$ & $174.8 m^{3}$ \\
$V_{a 2}$ & $174.8 m^{3}$ \\
\hline
\end{tabular}

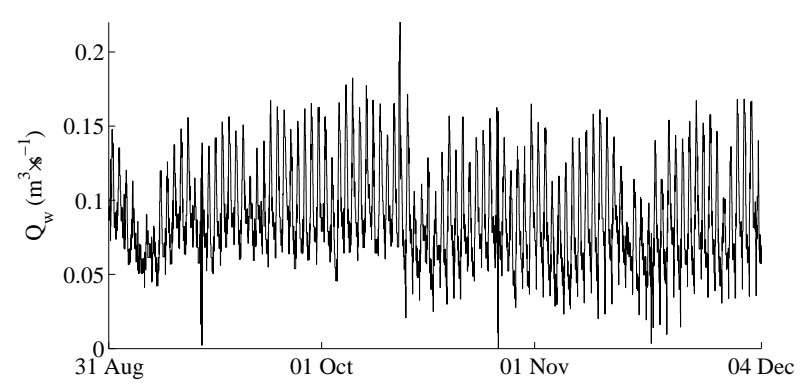

Fig. 3. The volumetric air flow rate from the patient wards to the animal rooms, which is calculated by taking the measured SCADA system values in $(m$. $s^{-1}$ ) and multiplying by the area of the duct. 
in Table II are ranges, the actual value used for the simulation still needs to be determined. For this simulation, the GUV irradiation decay constant is chosen to be the lowest value of its range, $k_{w}=$ 0.0987. However, to choose the quanta generation rate per infectious individual, $\phi_{w}$, and the incubation period, $\alpha$, within the literature plausible range, a parameter extraction problem is set up.

A least squares, cost function minimisation approach is used in the parameter extraction (Filter et al., 2005). This method involves the minimisation function solving the model (11) - (19) for a set of $\phi_{w}$ and $\alpha$ chosen by the minimisation function, then evaluating the cost of the solution. Through an iterative process, the minimisation function chooses a set of $\phi_{w}$ and $\alpha$ to minimise the cost.

The minimisation cost function is set up to be

$$
\begin{aligned}
J(\alpha, \phi) & =\sum_{j=1,2,3}\left(I_{1}\left(t_{j}\right)-I_{1, j}\right)^{2} \\
& +\sum_{j=1,2,3}\left(I_{2}\left(t_{j}\right)-I_{2, j}\right)^{2} \\
& +R \cdot\left(\mathcal{H}\left(\alpha-\alpha_{\max }\right) \cdot\left(\alpha-\alpha_{\max }\right)^{2}\right. \\
& +\mathcal{H}\left(\alpha_{\min }-\alpha\right) \cdot\left(\alpha_{\min }-\alpha\right)^{2} \\
& +\mathcal{H}\left(\phi-\phi_{\max }\right) \cdot\left(\phi-\phi_{\max }\right)^{2} \\
& \left.+\mathcal{H}\left(\phi_{\min }-\phi\right) \cdot\left(\phi_{\min }-\phi\right)^{2}\right)
\end{aligned}
$$

The cost function's first term describes the error between the simulation output, $I_{x}\left(t_{j}\right)$, and the TST results, $I_{x, j}$, of the number of infected in each animal room, $x$, for each of the TST measurements, $j$. The Heaviside function, $\mathcal{H}$, is used to penalise solutions that fall outside of the range allowed for the parameters, as given in Table II . The tuning factor, $R$, is used to penalise a solution that lies outside the constraint range. The tuning factor is set to $R=100$.

The Nelder-Mead simplex search function is used as a derivative-free based minimisation algorithm for this problem (Lagarias et al., 1998). The quanta generation rate per infectious individual for the simulations is found to be $\phi_{w}=2.5$ and the incubation period is $\alpha=0.03$.

\section{RESULTS AND DISCUSSION}

The simulation results, of the 31 August 2015 to 4 December 2015 experiment at the AIR facility, are given in Figure 6 and Figure 7, for the state variables of the guinea pig infected, exposed, and susceptible populations for each of the animal rooms and the amount of infectious particles in each of the three zones. The TST results of Table III , which fall within the period of the simulation, are also incorporated into Figure 6.

\subsection{Guinea pig population simulation results}

Guinea pigs have a similar disease progression as that of humans, with regards to $M$. tuberculosis; albeit, synonymous to the unsuccessful immune response of a human (Flynn, 2006; Dharmadhikari and Nardell, 2008). This makes guinea pigs well suited for airborne $M$. tuberculosis transmission studies as sentinel animals (Sakamoto, 2012). However, this measure of infectivity is problematic, because it does not account for the complexity and dynamics of disease manifestation in the guinea pig.

The exposed guinea pig population is an unmeasured state that attempts to account for some of the disease manifestation complexities and dynamics, by introducing an incubation period. This is an attempt at modelling the delay between infection by the pathogen to diagnosis through a visible induration from a TST. However, the exposed guinea

Table II . The parameters used for the simulation and their source. The parameters are defined in their most convenient units.

\begin{tabular}{llr}
\multicolumn{2}{l}{ Parameter } & \multicolumn{1}{c}{ Value } \\
\hline$\phi$ & $1.25-12.7$ quanta $\cdot h^{-1}$ & Nardell et al. (1991) \\
$p$ & $0.23 \mathrm{~m}^{3} \cdot h^{-1}$ & Riley et al. (1962) \\
$\theta$ & 1 quanta & Sze To and Chao (2010) \\
$\alpha$ & $0.03-0.2$ day $^{-1}$ & Ordway et al. (2007) \\
$k$ & $0.0987-0.472 \mathrm{~m}^{2} \cdot J^{-1}$ & Kowalski et al. (2000) \\
$H$ & $5 \mu W \cdot \mathrm{cm}^{-2}$ & Mphaphlele et al. (2015) \\
\hline
\end{tabular}

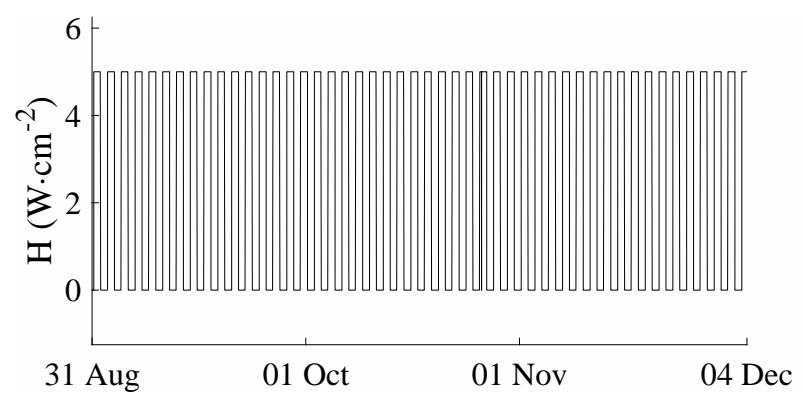

Fig. 4. The upper room GUV fixture fluence $H$ in the AIR facility. The GUV lamps were switched on during the days animal room one received ward air and were switched off during the days animal room two received ward air. 
Table III . Tuberculin skin test results for the two animal rooms. The total considered to have been infected per animal room is given by the sum of the number with positive TST results and the number euthanised.

\begin{tabular}{lcccc} 
& 20 Aug 2015 & 01 Oct 2015 & 30 Oct 2015 & 25 Nov 2015 \\
\hline Animal room 1 positive TST: & 0 & 0 & 9 & 11 \\
Animal room 1 euthanised: & 0 & 0 & 0 & 11 \\
\hline Animal room 1 total infected: & 0 & 0 & 9 & 22 \\
\hline Animal room 2 positive TST: & 0 & 0 & 2 & 5 \\
Animal room 2 euthanised: & 0 & 0 & 0 & 14 \\
\hline Animal room 2 total infected: & 0 & 0 & 2 & 19 \\
\hline
\end{tabular}
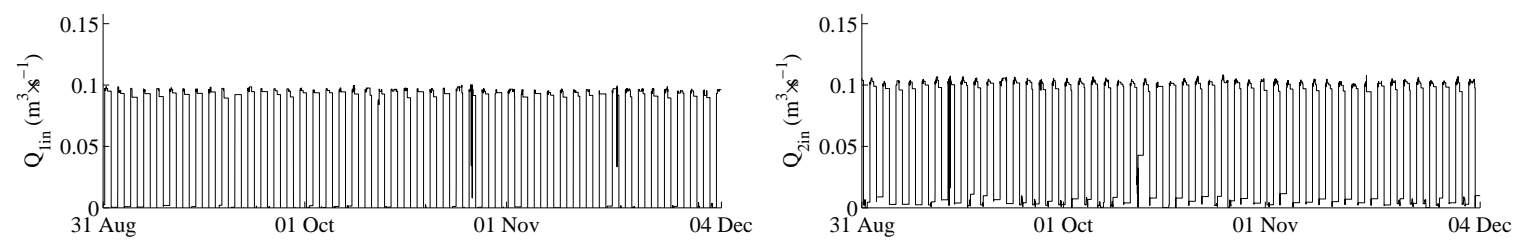

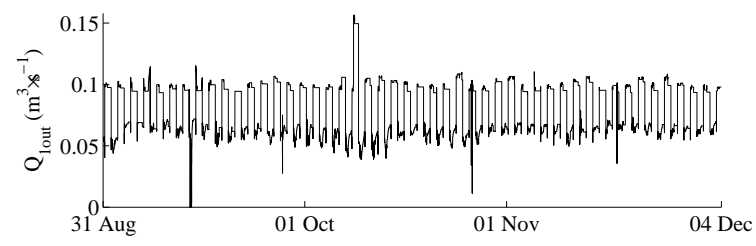

(a) Animal room one

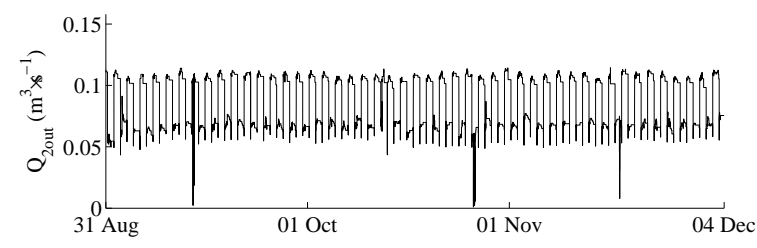

(b) Animal room two

Fig. 5. The volumetric air flow rates into the animal rooms $Q_{i \text { in }}$ (top) are calculated by taking the measured SCADA system airflow velocity $\left(m \cdot s^{-1}\right)$ and multiplying by the area of the duct. The volumetric air flow rates out of the animal rooms $Q_{i \text { out }}$ (bottom) are calculated by taking the ward air flow rate $Q_{i \text { in }}$ and adding the measurement of the HEPA filtered air flow rate into the animal room.

pig population result is theoretical, and should be considered with care.

\subsection{Infectious particle simulation results}

The number of infectious particles in the zones is an unmeasured, 'internal' state of the model. These states account for higher order dynamics in the prediction of the infected population, but their absolute value is theoretical, and should be considered as such. The unmeasured states of the quanta of infectious particles in the three zones is shown in Figure 7, and an arbitrary two day period is shown in Figure 8 to show the day to day pattern. Considerable fluctuations in the number of quanta in the zones can be noted from Figure 7, with three mechanisms attributed as responsible for these fluctuations.
The first mechanism that impacts the fluctuations in the number of infectious particles is the number of patients in the wards. This is clear from equation 17; however, can also be seen when comparing Figure 2 and 7. As an example, the average number of number of quanta on a day when the GUV lights are on can be compared during the first few days in October versus middle November. On 2 October 2015, six patients are in the ward and the average number of quanta is 3.8; however, on 15 November 2015, only two patients are in the ward and the average number of quanta is 1.4. The average airflow out of the ward is similar on the two days, being $0.0793 \mathrm{~m}^{3} \cdot \mathrm{s}^{-1}$ on 2 October 2015 and $0.0674 \mathrm{~m}^{3} \cdot \mathrm{s}^{-1}$ on 15 November 2015 .

The second mechanism that impacts the fluctuations of infectious particles in the zones is the ventilation rate. For the two animal rooms (Figure 
$5)$, this is expected to have a large impact on the fluctuations, due to the switching between ward air and clean HEPA filtered outside air on alternate days. When no ward air is supplied to the respective animal room, there is no source of infectious particles, and the clean air supply quickly removes the infectious particles that are in the animal room.

However, not only are the fluctuations of the air flow rate into the animal rooms significant, but so are the fluctuations in the airflow out of the wards. This airflow fluctuates due to day-night swings in temperature, because the feedback controller of the ward outlet fan was run in manual. The airflow fluctuation is especially noticeable on the simulated number of infectious particles in animal room two, as seen in Figure 8. The facility also experienced a number of power outages, due to an unstable supply from the national South African electrical grid during this period, causing the ventilation (and GUV fixtures) to switch off for the short span of time between the power outage and the time taken before the backup diesel generator switched in.

When the average ward air received by each of
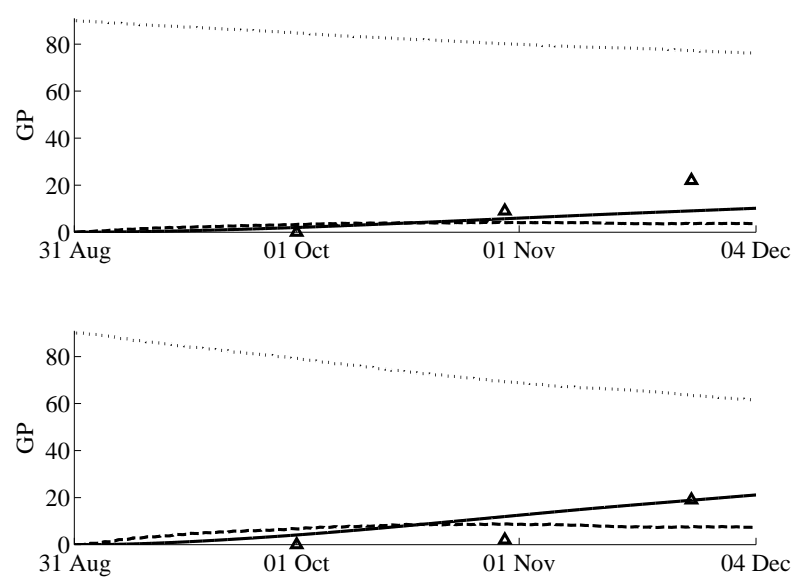

Fig. 6. The infected (solid), exposed (dashed) and susceptible (dotted) guinea pig populations in the AIR facility. The animal room one guinea pig populations (top) receive ward air during days when the upper room GUV fixtures are on, and animal room two guinea pig populations (bottom) receive ward air during the days when the upper room GUV fixtures are off. The total number of infected guinea pigs that were observed during the experiment (triangle), and as given in Table III the two animal rooms from Figure 5 is compared, it is found that animal room one gets $12.7 \%$ less ward air than animal room two. The average combined air into the animal rooms is $10.7 \%$ higher than the average measured air out of the ward. These deviations are most like a result of inaccuracies with the air flow measurement instruments, and give an idea of the typical uncertainty surrounding these measurements.

The third mechanism that impacts the fluctuations of infectious particles in the zones, is the upper room GUV irradiation. However, this fluctuation is intended from the experiment design. It can be seen
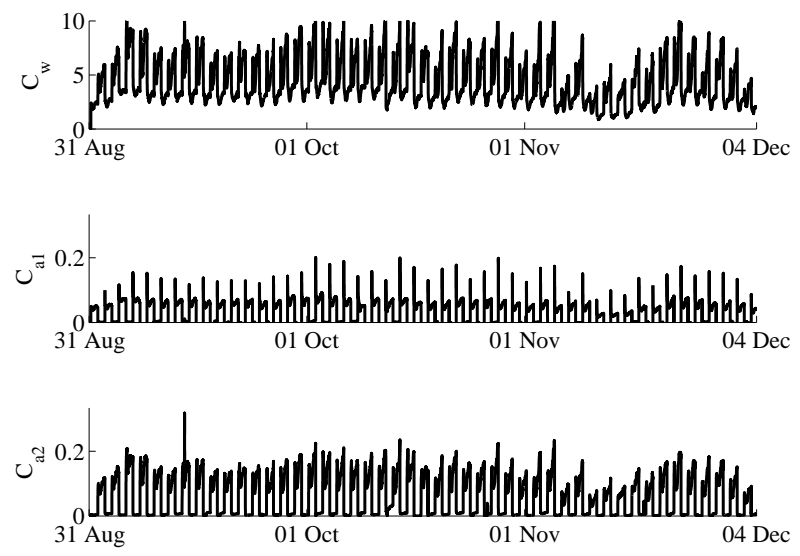

Fig. 7. The number of quanta $C_{w}$ (patient wards), $C_{a 1}$ (animal room one) and $C_{a 2}$ (animal room two) in each of the zones considered for the AIR facility.
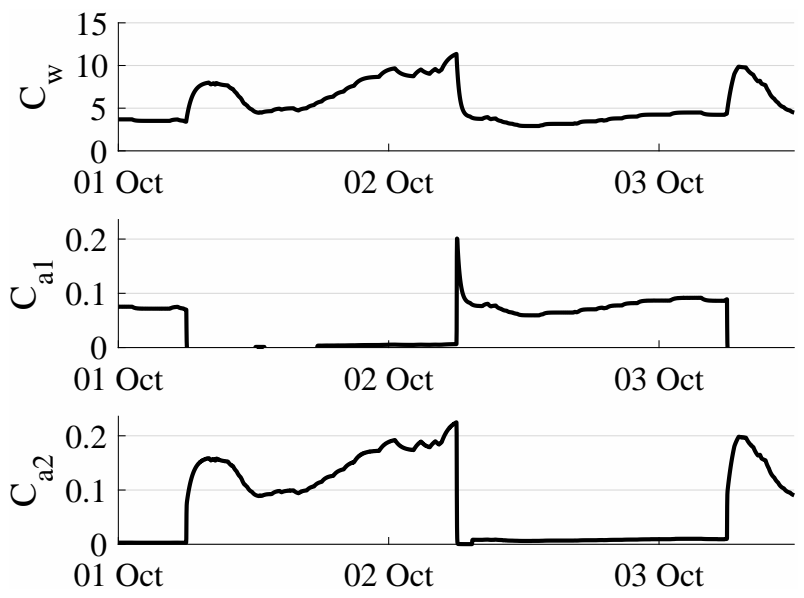

Fig. 8. The number of quanta $C_{w}$ (patient wards), $C_{a 1}$ (animal room one) and $C_{a 2}$ (animal room two) in each of the zones considered for the AIR facility. However, only a two day period is shown to highlight the day to day pattern seen in Figure 7. 
from Figures 7 and 8 that animal room one receives approximately half the number of infectious particles as opposed to what animal room two receives. This is due to the upper room GUV irradiation lowering the number of infectious particles in the wards on the days when it is on. This then also translates to fewer number of simulated infections in animal room one.

\subsection{Sensitivity of the simulation parameters}

There is significant uncertainty surrounding some of the parameters that were used in the simulation, which is mostly due to the large viable ranges of the parameters obtain from literature. To quantify the uncertainty, two sensitivity analyses were performed on the parameters used in the simulation. Both sensitivity analyses were conducted by keeping all parameters constant, and varying only a single parameter at a time.

The first sensitivity analysis aims to illustrate what the effects of uncertainty in the parameter values taken from literature are. The $\alpha, k$ and $\phi$ parameters were each varied by their range given in Table II , and the number of patients $I_{w}$ in the ward each day was increased and decreased by one. The resulting predictions for the number of infected guinea pigs in animal room one and animal room two can be seen in Figure 10. From this analysis, the impact of not knowing the true values of $\alpha, k$ and $\phi$ on the simulation outcomes is demonstrated. However, of greatest concern to the accuracy of simulation results is the quanta generation rate per infectious person parameter, $\phi$, which has the biggest impact on the simulation results for its plausible literature range. From the value used in the simulation, $\phi$ can vary by $458 \%$, which means the outcome of the simulation (the predicted number of infected guinea pigs) for animal room one can vary by $351 \%$ and for animal room two can vary by $256 \%$. However, model parameters that have been obtained through instrumentation measurements by the SCADA system are expected to have low uncertainty (on the order of $1-10 \%$ for typical instrumentation (Liptak, 2003)).

The second sensitivity analysis aims to illustrate the relative uncertainty in the different model parameters by increasing and decreasing each by an equal amount one at parameter at a time. The resulting predictions for the number of infected guinea pigs in animal room one and animal room two, when each parameter was arbitrarily increased and decreased by $50 \%$ while keeping the others constant, can be seen in Figure 9. From this analysis, it can be seen how the error of the true parameter value and the estimated value would affect the accuracy of the simulation results. It can also be noted that an uncertainty in some parameters is indistinguishable from an uncertainty in others, for example, the uncertainty in pulmonary respiration rate $p$, the quanta generation rate $\phi$ and the susceptibility to infection $\theta$ all affect the simulation outcome equally. This means that by estimating the quanta generation rate $\phi$, any error between the true and simulated values of the pulmonary respiration rate or susceptibility to infection, would result in a corresponding error between the true and simulated value of the quanta generation rate.

The sensitivity of the model to the quanta generation rate per infectious person parameter, $\phi$, indicates a research gap around the quanta of infection. Refining the constituent mechanism of the quanta of infection, can be beneficial in improving the predictability of simulations. This would also improve the understanding of factors that play a role in the mechanism of TB transmission. However, the quanta of infection unit of measure transparently represents this uncertainty, because it is a hypothetical unit. To refine the understanding of transmission and move away from the quanta of infection unit, would require additional measurements or other forms of experimental data (Strydom et al., 2017).

\subsection{Simulation fit and experiment outcome}

The difference between the simulated number of infected guinea pigs and the experimental results is shown in Figure 11. It can be seen that the simulated animal room one infected population better reproduces the experimental measurement of infected animals initially, but the simulated animal room two infected population is a much better fit for the end of the experiment.

The major mechanism that influences the difference between the simulated results of the two animal rooms, is the upper room GUV irradiation. Based on the parameters used, the simulation indicates that animal room one should have fewer infections than animal room two. However, animal room one actually had more infections than animal room two. This result is different to what was expected, both from the initiation of the experiment and from the simulation results.

To check whether there was enough contact time of the GUV irradiation with infectious particles, 

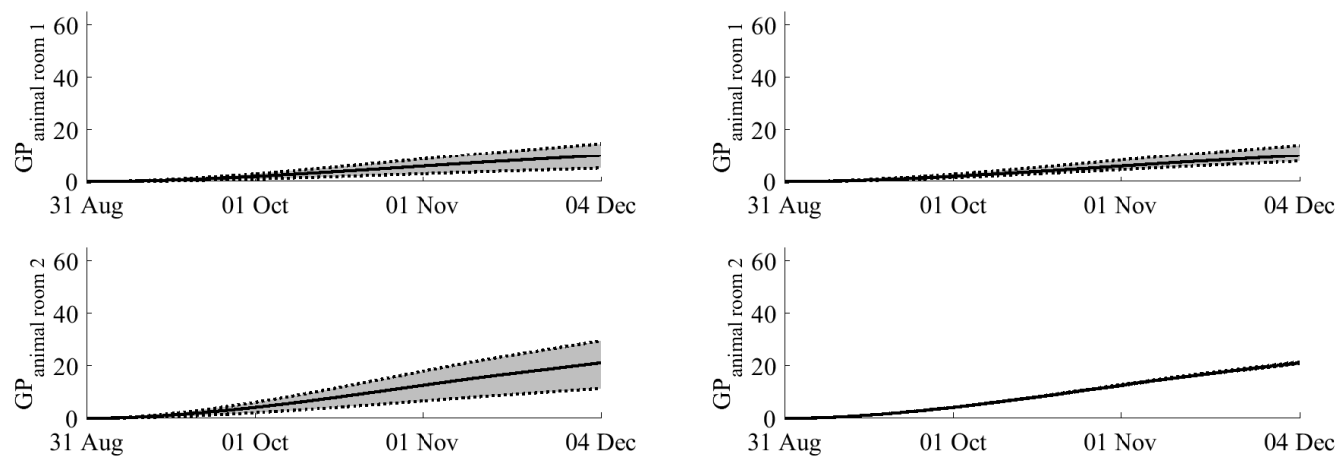

(a) $p, \phi$ and $\theta$

(b) $\alpha$
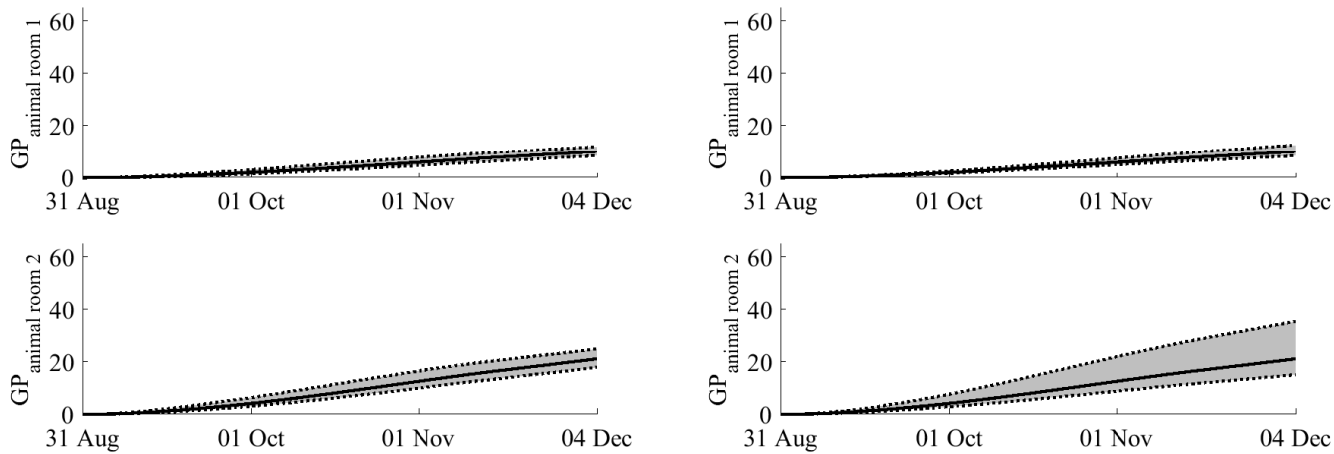

(c) $H$ and $k$

(d) $Q_{w}$
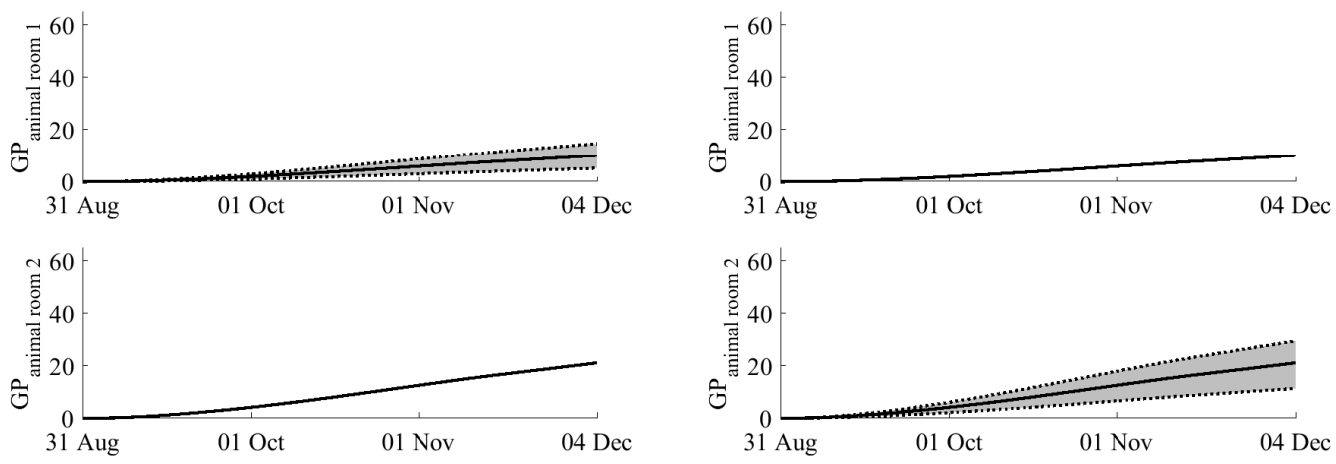

(e) $Q_{1 i n}$ and $Q_{1 o u t}$

(f) $Q_{2 i n}$ and $Q_{2 o u t}$

Fig. 9. The sensitivity of the number of infected when each of the parameters is varied by $\pm 50 \%$. The black line indicates the simulated number of infected, and the gray area the band of variation due to varying the parameter. Some of the parameters have the same effect on the number of infected, due to being factors of one another. The airflow $Q_{1 \text { in }}$ and $Q_{1 \text { out }}$ have a similar effect the results, but in different directions, giving the same overall band of uncertainty; $Q_{2 \text { in }}$ and $Q_{2 o u t}$ affect animal room two instead of animal room one.

considering the high air turnover rate of $57 h^{-1}$ due to the paddle fan, the time taken for infectious particles to decay will be compared to the time that air particles are expected to be in the upper room space.
Solving equation 10 gives that the time taken for infectious particles to decay is $\tau=\frac{1}{k_{i} H_{i}}$, which for this experiment was 203 seconds. While the time that air particles are expected to be in the upper room 

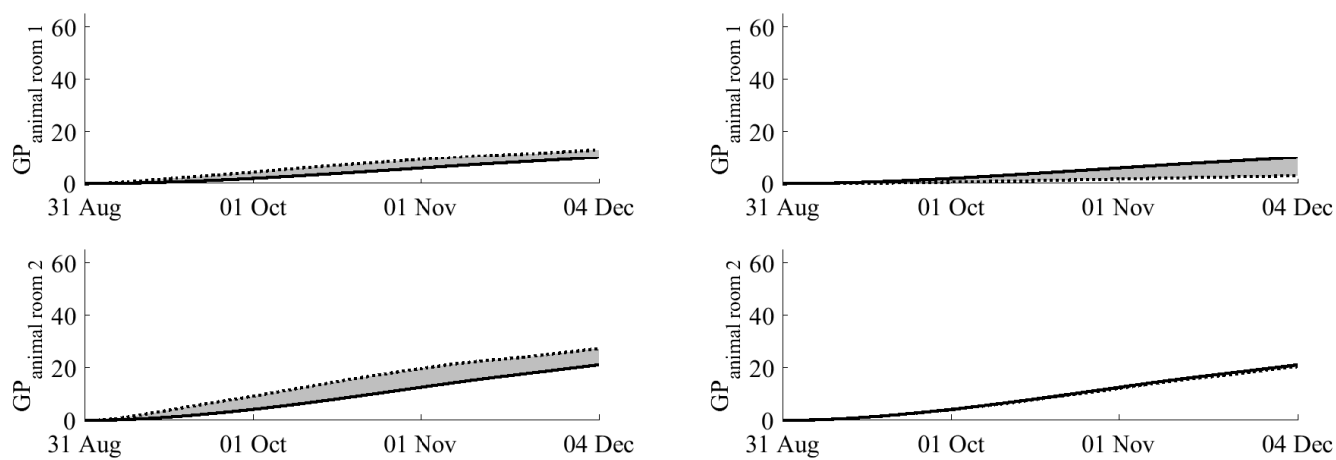

(a) $\alpha$

(b) $k$
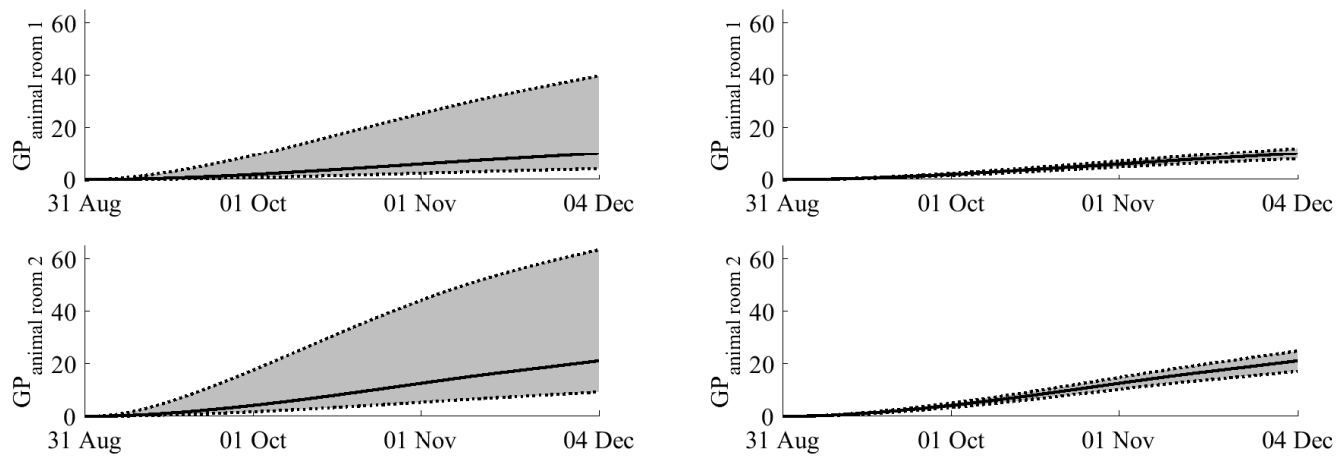

(c) $\phi$

(d) $I_{w}$

Fig. 10. The sensitivity of the number of infected when each of the parameters is varied by their literature given range, as in Table II . The black line indicates the simulated number of infected, and the gray area the band of variation. The number of patients in the ward, $I_{w}$, was varied by \pm 1 patient.

space is $\left(\frac{V_{U w}}{V_{w}} \cdot \text { air turnover rate }\right)^{-1}$, which for this experiment was 480 seconds. This means that on each circulation of air in the room, it is expected that $\left(1-e^{-480 / 203}\right)=90.6 \%$ of the infectious particles in that air will decay. Considering that approximately ten units of air are circulated per unit of air that is extracted from the room, it is concluded that there is sufficient contact time of the upper room GUV irradiation with the infectious particles.

A potential reason for the difference in expected to experimental results could be as a result of unmodelled dynamics of the disease manifestation. It could be that the actual number of infected in animal room two is under-represented by the last diagnosis. To remove some of the uncertainty regarding the incubation period, and unmodelled dynamics revolving around this, it is recommended that the sentinel animals be kept for another month after the end of the exposure to ward air. This has been done in a previous experiment (Dharmadhikari et al., 2011), and is now the standard that is used by the AIR facility.

Although the guinea pig and TST is still the most relevant clinical endpoint (Nardell and Sandin, 1999), a difficulty exists with using guinea pig TST results as the measurement for the number of infected. Because the TST cannot be conducted at higher frequency intervals, only three data points per animal room are available for this experiment. This makes it impossible to ensure that the data is a perfect representation of the actual trend. It is unknown whether data points are slightly skewed through the natural variation that results from slightly different responses to the exposure of the ward air by different animals. Using a large number of guinea pigs for an experiment does reduce the risk of the data not showing the true mean. Therefore, the AIR facility does try to ensure an adequate number 
of guinea pigs is used in the experiments conducted to give a representative sample of the number of infected.

The simulation results depict the expected results of fewer infections in animal room one well, as can be seen from Figure 6. This implies it is unlikely the fluctuations in airflow and the disruptions from power outages resulted in the experiment producing the unexpected results of more infections in animal room one. This is because the airflow rate data (Figures 3 and 5) and GUV switching data (Figure 4) were taken from the SCADA system. Therefore, the fluctuations in airflow and the disruptions from power outages were directly incorporated in the simulation outcome.

The simulation also indicates the dynamic effects of the GUV lamp switching on the infectious particles is much faster than the switching cycle. This can be seen from Figures 7 and 8, where there appears to be a spike in the infectious particles entering animal room one whenever ward air is switched to this animal room. These spikes are caused by the time taken by the GUV lamps to reduce the number of infectious particles in the ward, and hence the rate of infectious particles transferred to animal room one. This implies the unexpected experiment results are unlikely to be caused by the change in steady-state of the infectious particles in the ward that happens after switching the GUV lamps.

Based on these observations, it is the opinion of the authors, that the unexpected experimental results were not caused due to negligence on the part of the AIR facility. The phenomenon observed

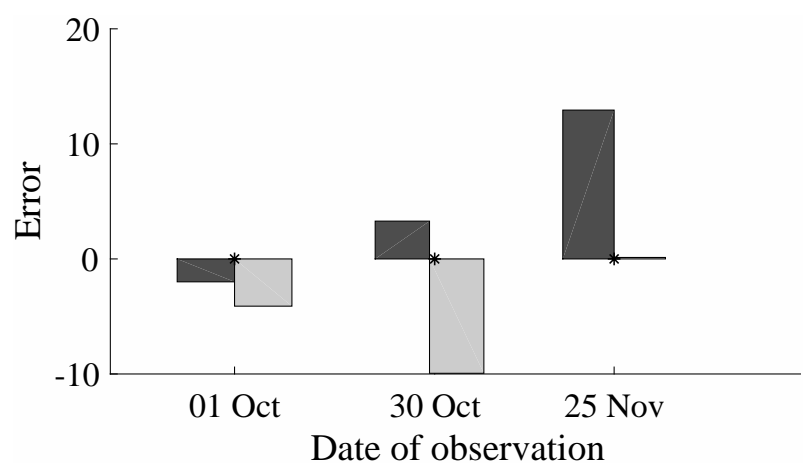

Fig. 11. The error of the prediction giving by the difference between the number of infected from the experiment and the simulated results. The left bars indicate the difference for animal room one, and the right bars for animal room two. is not yet understood, and will require further investigation.

\section{CONCLUSION}

The given framework description on the risk of transmission of an airborne infectious disease in indoor spaces is modular in nature. This makes the modelling process simpler through the presentation of different mechanisms and how these mechanisms can be combined to describe different situations. The given approach was demonstrated through the simulation of an experiment, conducted at the AIR facility from 31 August 2015 to 4 December 2015.

The simulation helped investigate potential reasons for the experiment not producing the results that were expected. Although the cause of the experiment not producing expected results was not ascertained, it was found that it was not due to an oversight on the part of the AIR facility. This indicates that there is some mechanism or dynamic surrounding the risk of transmission of tuberculosis that is not yet fully understood, and requires further research. The sensitivity analysis further shows that accurate simulation predictions are challenging to achieve when using the quanta of infection unit, due to very large uncertainty surrounding the quanta generation rate per infector.

\section{ACKNOWLEDGMENT}

The financial assistance of the National Research Foundation (DAAD-NRF) towards this research is hereby acknowledged. Opinions expressed and conclusions arrived at, are those of the author and are not necessarily to be attributed to the DAADNRF.

\section{REFERENCES}

Antsaklis, P. (1994). Defining intelligent control. IEEE Control Systems Magazine, 4(5):58-66.

Beggs, C. B., Donnelly, J. K., Kerr, K. G., Sleigh, P. A., Mara, D. D., and Cairns, G. (2000). The use of engineering controls to disinfect Mycobacterium tuberculosis and airborne pathogens in hospital buildings. Indoor and Built Environment, 9(1):17-27, doi: 10.1177/1420326×0000900106. Beggs, C. B., Noakes, C. J., Sleigh, P. A., Fletcher, L. A., and Siddiqi, K. (2003). The transmission of tuberculosis in confined spaces: An analytical review of alternative epidemiological models. The International Journal of Tuberculosis and Lung Disease, 7(11):1015-1026.

Bhangar, S., Huffman, J. A., and Nazaroff, W. W. (2014). Size-resolved fluorescent biological aerosol particle concen- 
trations and occupant emissions in a university classroom. Indoor Air, 24(6):604-617, doi: 10.1111/ina.12111.

Chapman, S. and Cowling, T. G. (1970). The mathematical theory of non-uniform gases: an account of the kinetic theory of viscosity, thermal conduction and diffusion in gases. Cambridge University Press.

Craig, I. K. and Xia, X. (2005). Can HIV/AIDS be controlled? Applying control engineering concepts outside traditional fields. IEEE Control Systems Magazine, 25(1):80-83, doi: 10.1109/MCS.2005.1388805.

David, H. L. (1973). Response of Mycobacteria to ultraviolet light radiation. The American Review of Respiratory Disease, 108(5):1175-1185.

Dharmadhikari, A. S., Basaraba, R. J., Van Der Walt, M. L., Weyerd, K., Mphahlele, M., Venter, K., Jensen, P. A., First, M. W., Parsons, S., McMurray, D. N., Orme, I. M., and Nardell, E. A. (2011). Natural Infection of guinea pigs exposed to patients with highly drugresistant tuberculosis. Tuberculosis, 91(4):329-338, doi: $10.1016 / j$.tube .2011 .03 .002 .

Dharmadhikari, A. S., Mphahlele, M., Stoltz, A., Venter, K., Mathebula, R., Masotla, T., Lubbe, W., Pagano, M., First, M., Jensen, P. A., Van Der Walt, M., and Nardell, E. A. (2012). Surgical face masks worn by patients with multidrug-resistant tuberculosis: Impact on infectivity of air on a hospital ward. American Journal of Respiratory and Critical Care Medicine, 185(10):11041109, doi: 10.1164/rccm.201107-11900C.

Dharmadhikari, A. S., Mphahlele, M., Venter, K., Stoltz, A., Mathebula, R., Masotla, T., Van Der Walt, M., Pagano, M., Jensen, P., and Nardell, E. (2014). Rapid impact of effective treatment on transmission of multidrug-resistant tuberculosis. International Journal of Tuberculosis and Lung Disease, 18(9):1019-1025, doi: 10.5588/ijtld.13.0834.

Dharmadhikari, A. S. and Nardell, E. A. (2008). What animal models teach humans about tuberculosis. American Journal of Respiratory Cell and Molecular Biology, 39(5):503-508, doi: 10.1165/rcmb. 2008-0154TR.

Doyle, F., Jovanovic, L., Seborg, D., Parker, R. S., Bequette, B. W., Jeffrey, A. M., Xia, X., Craig, I. K., and McAvoy, T. (2007). A tutorial on biomedical process control. Journal of Process Control, 17:571-572, doi: 10.1016/j.jprocont.2007.01.012.

du Toit, E. F. and Craig, I. K. (2015). Selective pinning control of the average disease transmissibility in an HIV contact network. Physical Review E, 92(1), doi: 10.1103/PhysRevE. 92.012810.

Dye, C., Glaziou, P., Floyd, K., and Raviglione, M. (2013). Prospects for tuberculosis elimination. Annual Review of Public Health, 34:271-86, doi: 10.1146/annurev-publhealth-031912-114431.

Ehrenkranz, N. J. and Kicklighter, J. L. (1972). Tuberculosis outbreak in a general hospital: evidence for airborne spread of infection. Annals of Internal Medicine, 77:377-382.

Escombe, A. R., Oeser, C., Gilman, R. H., Navincopa, M., Ticona, E., Martínez, C., Caviedes, L., Sheen, P., Gonzalez, A., Noakes, C., Moore, D. A. J., Friedland, J. S., and Evans, C. A. (2007). The detection of airborne transmission of tuberculosis from HIV-infected patients, using an in vivo air sampling model. Clinical Infectious Diseases, 44(10):13491357, doi: 10.1086/515397.

Fennelly, K. P., Martyny, J. W., Fulton, K. E., Orme, I. M., Cave, D. M., and Heifets, L. B. (2004). Coughgenerated aerosols of Mycobacterium tuberculosis: a new method to study infectiousness. American Journal of Respiratory and Critical Care Medicine, 169(5):604-609, doi: $10.1164 / \mathrm{rccm} .200308-11010 \mathrm{C}$.

Fiegel, J., Clarke, R., and Edwards, D. A. (2006). Airborne infectious disease and the suppression of pulmonary bioaerosols. Drug Discovery Today, 11(1):51-57, doi: 10.1016/S1359-6446(05) 03687-1.

Filter, R., Xia, X., and Gray, C. (2005). Dynamic HIV/AIDS parameter estimation with application to a vaccine readiness study in southern Africa. IEEE Transactions on Biomedical Engineering, 52(5):784-791, doi: 10.1109/TBME. 2005.844274.

Fisk, W. J., Seppanen, O., Faulkner, D., and Huang, J. (2004). Economic benefits of an economizer system: Energy savings and reduced sick leave. Technical report, Lawrence Berkeley National Laboratory.

Flynn, J. L. (2006). Lessons from experimental Mycobacterium tuberculosis infections. Microbes and Infection, 8(4):1179-1188, doi: 10.1016/j.micinf.2005.10.033.

Gammaitoni, L. and Nucci, M. C. (1997). Using a mathematical model to evaluate the efficacy of TB control measures. Emerging Infectious Diseases, 3(3):335-342, doi: 10.3201/eid0303.970310.

Jeffrey, A. M., Xia, X., and Craig, I. K. (2003). When to initiate HIV therapy: A control theoretic approach. IEEE Transactions on Biomedical Engineering, 50(11):12131220, doi: 10.1109/TBME. 2003.818465

Jones-López, E. C., Namugga, O., Mumbowa, F., Ssebidandi, M., Mbabazi, O., Moine, S., Mboowa, G., Fox, M. P., Reilly, N., Ayakaka, I., Kim, S., Okwera, A., Joloba, M., and Fennelly, K. P. (2013). Cough aerosols of Mycobacterium tuberculosis predict new infection: A household contact study. American Journal of Respiratory and Critical Care Medicine, 187(9):1007-1015.

Karim, S. S. A., Churchyard, G. J., Karim, Q. A., and Lawn, S. D. (2009). HIV infection and tuberculosis in South Africa: an urgent need to escalate the public health response. The Lancet, 374(9693):921-933, doi: 10.1016/S0140-6736 (09) 60916-8.

King, M.-F., Noakes, C., Sleigh, P., and Camargo-Valero, M. (2013). Bioaerosol deposition in single and twobed hospital rooms: A numerical and experimental study. Building and Environment, 59:436-447, doi: 10.1016/j. buildenv.2012.09.011.

Kowalski, W. J., Bahnfleth, W. P., Witham, D. L., Severin, B. F., and Whittam, T. S. (2000). Mathematical modeling of ultraviolet germicidal irradiation for air disinfection. Quantitative Microbiology, 2(3):249-270, doi: 10.1023/A: 1013951313398

Lagarias, J. C., Reeds, J. A., Wright, M. H., and Wright, P. E. (1998). Convergence Properties of the Nelder-Mead Simplex Method in Low Dimensions. SIAM Journal on Optimization, 9(1):112-147, doi: 10.1137/S1052623496303470. $\mathrm{Li}$, X., Inthavong, K., Ge, Q., and Tu, J. (2013). Numerical investigation of particle transport and inhalation using standing thermal manikins. Building and Environment, 60:116-125, doi: 10.1016/j.buildenv.2012.11.014.

Li, Y., Leung, G. M., Tang, J. W., Yang, X., Chao, C. Y. H., Lin, J. Z., Lu, J. W., Nielsen, P. V., Niu, J., Qian, H., Sleigh, A. C., Su, H. J. J., Sundell, J., Wong, T. W., and Yuen, P. L. (2007). Role of ventilation in airborne transmission of infectious agents in the built environment A multidisciplinary systematic review. Indoor Air, 17(1):218, doi: $10.1111 / \mathrm{j} .1600-0668.2006 .00445 . \mathrm{x}$.

Liptak, B. G. (2003). Instrument Engineers' Handbook, Volume One: Process Measurement and Analysis. CRC press.

Lönnroth, K., Castro, K. G., Chakaya, J. M., Chauhan, L. S., Floyd, K., Glaziou, P., and Raviglione, M. C. (2010). Tuberculosis control and elimination 2010-50: cure, care, and social development. The Lancet, 375(9728):1814-1829, doi: 10.1016/S0140-6736(10)60483-7.

Miller, S. L. and MacHer, J. M. (2000). Evaluation of a Methodology for Quantifying the Effect of Room 
Air Ultraviolet Germicidal Irradiation on Airborne Bacteria. Aerosol Science and Technology, 33(3):274-295, doi: $10.1080 / 027868200416259$.

Mousavi, E. S. and Grosskopf, K. R. (2014). Ventilation Rates and Airflow Pathways in Patient Rooms: A Case Study of Bioaerosol Containment and Removal. Annals of Occupational Hygiene, 59(9):1190-1199, doi: 10.1093/annhyg/mev048.

Mphaphlele, M., Dharmadhikari, A. S., Jensen, P. A., Rudnick, S. N., Van Reenen, T. H., Pagano, M. A., Leuschner, W., Sears, T. A., Milonova, S. P., Van Der Walt, M., Stoltz, A. C., Weyer, K., and Nardell, E. A. (2015). Institutional tuberculosis transmission: Controlled trial of upper room ultraviolet air disinfection: A basis for new dosing guidelines. American Journal of Respiratory and Critical Care Medicine, 192(4):477-484, doi: $10.1164 / \mathrm{rccm} .201501-00600 \mathrm{C}$

Mupere, E., Schiltz, N. K., Mulogo, E., Katamba, A., Nabbuye-Sekandi, J., and Singer, M. E. (2013). Effectiveness of active case-finding strategies in tuberculosis control in Kampala, Uganda. International Journal of Tuberculosis and Lung Disease, 17(2):207-213, doi: 10.5588/ijtld. 12.0160 .

Nardell, E. and Dharmadhikari, A. S. (2010). Turning off the spigot: reducing drug-resistant tubervulosis transmission in resource-limited settings. International Journal of Tuberculosis and Lung Disease, 14(10):1233-1243.

Nardell, E. A. (2004). Catching droplet nuclei: toward a better understanding of tuberculosis transmission. American Journal of Respiratory and Critical Care Medicine, 169(5):553-4, doi: 10.1164/rccm. 2401003.

Nardell, E. A. (2016). Indoor environmental control of tuberculosis and other airborne infections. Indoor Air, 26(1):79-87, doi: 10.1111/ina.12232.

Nardell, E. A., Keegan, J., Cheney, S. A., and Etkind, S. C. (1991). Airborne infection: Theoretical limits of protection achievable by building ventilation. American Review of Respiratory Disease, 144(2):302-306, doi: 10.1164/ajrccm/144.2.302.

Nardell, E. A. and Sandin, R. L. (1999). Air sampling for tuberculosis - Homage to the lowly guinea pig. Chest, 116(4):1143-1145, doi: 10.1378/chest.116.4.1143.

Nazaroff, W. W., Nicas, M., and Miller, S. L. (1998). Framework for evaluating measures to control nosocomial tuberculosis transmission. Indoor Air, 8(4):205-218, doi: $10.1111 / j .1600-0668.1998 .00002 . x$.

Nicas, M. (1996a). An analytical framework for relating dose, risk, and incidence: An application to occupational tuberculosis infection. Risk Analysis, 16(4):527-538, doi: 10.1111/j.1539-6924.1996.tb01098.x.

Nicas, M. (1996b). Estimating exposure intensity in an imperfectly mixed room. American Industrial Hygiene Association Journal, 57(6):542-550, doi: $10.1080 / 15428119691014756$.

Nicas, M. (2000). Markov modeling of contaminant concentrations in indoor air. American Industrial Hygiene Association Journal, 61(4):484-491, doi: $10.1080 / 15298660008984559$.

Nicas, M., Nazaroff, W. W., and Hubbard, A. (2005). Toward understanding the risk of secondary airborne infection: Emission of respirable pathogens. Journal of Occupational and Environmental Hygiene, 2(3):143-54, doi: $10.1080 / 15459620590918466$.

Nicas, M. and Seto, E. (1997). A simulation model for occupational tuberculosis transmission. Risk Analysis, 17(5):609-16, doi: 10.1111/j.1539-6924.1997.tb00901.x.

Noakes, C. J., Beggs, C. B., Sleigh, P. A., and Kerr, K. G. (2006). Modelling the transmission of airborne infections in enclosed spaces. Epidemiology and Infection, 134:1082-
1091, doi: $10.1017 /$ S0950268806005875

Noakes, C. J. and Sleigh, P. A. (2008). Applying the WellsRiley equation to the risk of airborne infection in hospital environments: The importance of stochastic and proximity effects. In Indoor Air 2008: The 11th International Conference on Indoor Air Quality and Climate, pages 1722, Copenhagen.

Ordway, D., Palanisamy, G., Henao-Tamayo, M., Smith, E. E., Shanley, C., Orme, I. M., and Basaraba, R. J. (2007). The cellular immune response to Mycobacterium tuberculosis infection in the guinea pig. Journal of Immunology, 179(4):2532-2541, doi: 10.4049/jimmunol.179.4.2532.

Riley, E. C., Murphy, G., and Riley, R. L. (1978). Airborne spread of measles in a suburban elementary school. American Journal of Epidemiology, 107(5):421-432.

Riley, R., Mills, C., Nyka, W., Weinstock, N., Storey, P., Sultan, L., Riley, M., and Wells, W. (1959). Aerial dissemination of pulmonary tuberculosis: a two-year study of contagion in a tuberculosis ward. American Journal of Hygiene, 70(2):185-196.

Riley, R. L. (1974). Airborne infection. American Journal of Medicine, 57(3):466-475.

Riley, R. L. (2001). What nobody needs to know about airborne infection. American Journal of Respiratory and Critical Care Medicine, 163(1):7-8, doi: 10.1164/ajrccm.163.1.hh11-00.

Riley, R. L., Knight, M., and Middlebrook, G. (1976). Ultraviolet susceptibility of BCG and virulent tubercle bacilli. American Review of Respiratory Disease, 113(4):413-418. Riley, R. L., Mills, C. C., O'Grady, F., Sultan, L. U., Wittstadt, F., and Shivpuri, D. N. (1962). Infectiousness of Air from a Tuberculosis Ward. American Review of Respiratory Disease, 85(4):511-525.

Sakamoto, K. (2012). The Pathology of Mycobacterium tuberculosis Infection. Veterinary Pathology, 49(3):423439, doi: 10.1177/0300985811429313.

Schwander, S. K. and Ellner, J. (2006). The human host: immunology and susceptibility. In Raviglione, M. C. and Lambregts, C. S., editors, Reichman and Hershfield's Tuberculosis, Part A: A Comprehensive, International Approach, chapter 6, pages 117-154. CRC Press, London, 3rd edition.

Shampine, L. F. (1994). Numerical solution of ordinary differential equations, volume 4. CRC Press.

Shampine, L. F. and Reichelt, M. W. (1997). The MATLAB ODE Suite. SIAM Journal on Scientific Computing, 18(1):1-22, doi: 10.1137/S1064827594276424.

Strydom, D., Küsel, R. R., and Craig, I. K. (2017). When is it appropriate to model transmission of tuberculosis using a dose response model? IFAC-PapersOnLine, 50(2):31-36, doi: $10.1016 / \mathrm{j}$. ifacol.2017.12.006.

Sze To, G. N. and Chao, C. Y. H. (2010). Review and comparison between the Wells-Riley and doseresponse approaches to risk assessment of infectious respiratory diseases. Indoor Air, 20(1):2-16, doi: $10.1111 / \mathrm{j} .1600-0668.2009 .00621 . \mathrm{x}$.

Tang, J. W., Li, Y., Eames, I., Chan, P. K. S., and Ridgway, G. L. (2006). Factors involved in the aerosol transmission of infection and control of ventilation in healthcare premises. Journal of Hospital Infection, 64(2):100-114, doi: 10.1016/j.jhin.2006.05.022.

Wells, W. F. (1955). Airborne Contagion and Air Hygiene. Cambridge University Press, Cambridge.

World Health Organization (2004). Laboratory biosafety manual. Geneva, 3 edition.

Xia, X. (2003). Estimation of HIV/AIDS parameters. Automatica, 39(11):1983-1988, doi: 10.1016/S0005-1098(03) 00220-6.

Xia, X. and Moog, C. H. (2003). Identifiability of nonlinear 
systems with application to HIV/ AIDS models. IEEE Transactions on Automatic Control, 48(2):330-336, doi: 10.1109/TAC. 2002.808494.

Yang, S., Lee, G. W. M., Chen, C.-M., Wu, C.-C., and Yu, K.P. (2007). The size and concentration of droplets generated by coughing in human subjects. Journal of Aerosol Medicine, 20(4):484-494, doi: 10.1089/jam.2007.0610.

Yates, T. A., Khan, P. Y., Knight, G. M., Taylor, J. G., McHugh, T. D., Lipman, M., White, R. G., Cohen, T., Cobelens, F. G., Wood, R., Moore, D. A. J., and Abubakar, I. (2016). The transmission of Mycobacterium tuberculosis in high burden settings. The Lancet Infectious Diseases, 16(2):227-238, doi: 10.1016/S1473-3099(15) 00499-5.

Yuen, C. M., Amanullah, F., Dharmadhikari, A. S., Nardell, E. A., Seddon, J. A., Vasilyeva, I., Zhao, Y., Keshavjee, S., and Becerra, M. C. (2015). Turning off the tap: Stopping tuberculosis transmission through active case-finding and prompt effective treatment. The Lancet, 386(10010):23342343, doi: 10.1016/S0140-6736(15)00322-0. 